\title{
SHORTENED VERSIONS OF THE INTERPERSONAL SENSITIVITY MEASURE, PARENTAL BONDING INSTRUMENT AND INTIMATE BOND MEASURE
}

\author{
A. L. TODD, ' P. M. BOYCE.' A. C. HEATH' and N. G. MARTTN ${ }^{3}$ \\ 'Department of Psychiatry, University of Sydney, Nepean Hospital. Kingswood NSW 2751, Australia, \\ 'Department of Psychiatry, Washington University School of Medicine. St Louis. MO 63110, U.S.A. and \\ ${ }^{3}$ Queensiand Institute of Medical Research. Brisbane QLD 4029, Australia
}

(Received 10 November 1992; received for publication 7 July 1993)

\begin{abstract}
Summary-A questionnaire sent in 1990 to over 2000 pairs of 18 to 26-year-old twins included shortened versions of the Interpersonal Sensitivity Measure (IPSM). Parental Bonding Instrument (PBI) and Intimate Bond Measure (IBM). To evaluate the vaiidity of these short seajes. and investigate their stability over time, full versions of the questionnaires were mailed 1 year later, in 1991, to a subsample of 100 twin pairs ( 25 monozygotic females, 25 monozygotic maies. 25 dizygotic females, 25 dizygotic males). After follow-up, replies were received from 51 male and 70 female individuais (60\% response overall). From the full length questionnaires used in 1991 we were able to generate test scores for both the shortened and full scale versions, whereas from the 1990 study only short scale scores were available. Correlations between the shortened and full length scales obtained in 1991 were ingh $(r=0.64-0.99)$. Correlations between test scores on the shortened versions of the instruments in 1990 and 1991 were moderate $(r=0.47-0.55)$ for the IPSM, and siightly higher for the shortened PBI scajes $(0.55-0.66)$ and the shortened IBM care subscale $(r=0.58)$. Correiations between the 1990 shortened scales with their full length 1991 counterparts were slightly lower for the IPSM $(r=0.31-0.50)$, comparable or slightly higher for the PBI $(r=0.61-0.70)$, and the same for the IBM $(r=0.58)$. Internal reliabilities of the shortened test versions were slightly reduced but satisfactory in most cases when compared with the full instruments: Cronbach's alpha full length $1991 \quad z=0.81-0.96$; shortened $1991 \quad z=0.46-0.96$; shortened 1990 $\alpha=0.48-0.96$. We conclude that some information is lost using the shortened versions of these instruments, and there is also some change over time. However, the relationsinip between the tests, using either the full length versions or the shortened counterparts. remained constant suggesting that the shortened versions can be used in epidemiological studies.
\end{abstract}

\section{INTRODUCTION}

In recent years there has been considerable interest in the relationship between attachment theory and neurotic illness. Early parental attachments and current intimate reiationships have been linked theoretically to the genesis of adult depression (Bowlby. 1988). Empirical evidence supports such an association. Henderson, Duncan-Jones. McAuley and Ritchie (1978) have shown that deficiencies in attachments or social bonds increase the risk of developing depression. In contrast, good social support may act as a buffer against neurotic decompensation following adverse life events (Alloway \& Bebbington, 1987).

Three self-report questionnaires have been developed to measure specific aspects of interpersonal relationships which may be risk factors to the onset of neurotic illness. The Parental Bonding Instrument (PBI; Parker, Tupling \& Brown. 1979) assesses an adult's recollection of early parental bonding and attachment difficulties on dimensions of care and overprotection. The Intimate Bond Measure (IBM; Wilhelm \& Parker, 1988) measures a person's perception of current intimate relationships using two factors, care and control. The Interpersonal Sensitivity Measure (IPSM; Boyce \& Parker, 1989) is a scale designed to measure depression-prone personality style. It identifies individuals with an undue or excessive awareness of. and sensitivity to. the behaviour and feelings of others (Boyce \& Parker, 1989). The IPSM comprises five subscales: interpersonal awareness, separation anxiety, need for approval. timidity. and fragile inner-seif. While the PBI. IBM and IPSM all measure aspects of interpersonal relationships. intercorrelations between the instruments are low, suggesting the underlying constructs are independent (Boyce. Hickie \& Parker, 1991). 
A number of studies have demonstrated that dysfunctional parenting, dysfunctional intimate relationships, and high interpersonal sensitivity are vulnerability factors to non-meianchoilc depression. It has been suggested that these risk factors impart their deleterious effects principally by their ability to lower self-esteem (Boyce et al., 1991). While a complete review of these studies is beyond the scope of this paper specific findings will be highlighted. Patients with non-melancholic depression are more likely to report exposure to dysfunctional parenting (especially 'affectionless control') than both non-depressive controls and patients with endogenous or bipolar depressive disorders (Parker, 1983a, b; Parker, Kiloh \& Hayward, 1987; Plantes, Prusoff, Brennan \& Parker, 1988; Hickie, Wilhelm, Parker, Boyce, Hadzi-Pavlovic, Brodaty \& Mitchell, 1990). Subjects with non-melancholic depression are also more likely to report a current intimate partner as being less caring and overcontrolling than controls (Hickie et al., 1990). Finally, non-melancholic depressives are more interpersonally sensitive than melancholic depressives (Boyce, Parker, Hickie, Wilhelm, Brodaty \& Mitchell, 1990), and women with high interpersonal sensitivity have been found to be at increased risk of developing depression following childbirth (Boyce et al., 1991).

Recent studies in behavioural genetics using twin samples have explored the genetic component of such vulnerability traits (Cadoret, O'Gorman, Heywood \& Troughton, 1985; Martin, Jardine, Andrews \& Heath, 1988; Andrews, Stewart, Allen \& Henderson, 1990; Kendler, Neale, Kessler, Heath \& Eaves, 1992). Methodologically, the combined use of psychological questionnaires such as the PBI, IBM and IPSM is often hindered by their length. The present study examined whether shorter versions of these scales are valid measures of the underlying constructs captured by the full length instruments. We also assessed the stability of test scores over time. If the medium term stability is only moderate then one might conclude that the constructs refiect the state of current well-being. If they are high, on the other hand, they may be measuring genuinely stable aspects of a person's environment and/or their personality.

\section{METHOD}

\section{Subjects}

Ss were twins drawn from the Australian NH\&MRC Twin Registry (Jardine, Martin \& Henderson, 1984). Twins are enrolled on the Registry voluntarily and most were recruited while at school. $S \mathrm{~s}$ in the present study were all twins aged 18-26 years, who completed and returned a 16-page questionnaire booklet mailed to them in May 1990. Complete replies were received from about 2000 twin pairs.

The $S s$ were part of a larger project, whose primary focus was the study of alcohol use and abuse. The questionnaire booklet canvassed responses to a range of psychological, social, demographic and health measures. Data collection in this larger project is not yet complete and full details will be published elsewhere.

Included in the questionnaire booklet were items from the IPSM, PBI and IBM. There were many competing demands for inclusion in the questionnaire booklet and it was not possible to use full versions of all instruments. Therefore authors of instruments were asked to supply shortened versions.

\section{Instruments}

The full IPSM (Boyce \& Parker, 1989) contains 36 items. Twelve items were selected on the basis of their factor loadings on the IPSM and the internal consistency of the subscales when items were deleted. The shortened form included 4 items each for the 'interpersonal awareness' and 'separation anxiety' subscales, and 2 items each for the 'timidity' and 'fragile inner-self suoscales. No items were included in the shortened form for the 'need for approval' subscale. since this had poor internal reliability in the original analysis and its loadings on the general factor were low. In the 1990 study these 12 items were mixed randomly among 48 items of the short form of the Revised Eysenck Personality Questionnaire (EPQ-R-S: Eysenck, Eysenck \& Barrett. 1985) and a further 7 supplementary impulsiveness items. For consistency of response set. the IPSM items were slightly reworded and the response options changed from the original 4-point Likert scale to 2 categories.

The full PBI (Parker et al., 1979) contains a 'care' dimension defined by 12 items and an 
'overprotection' dimension deñned by 13 items. and Ss completed a PBI for each parent. Seven items from the PBI were used in the shortened version. comprising 3 care items and 4 overprotection items. The four-point response set was retained although the wording for the categories was slightly altered.

The original IBM (Wilhelm \& Parker. 1988) has 24 items. with 12 items each measuring 2 factors 'care' and 'control'. Ten items from the care subscale. which were considered to be the most relevant as risk factors for neurotic illness, were selected for inciusion in the 1990 study.

\section{Procedure}

The rationale for this study is the concern that the shortened versions of the instruments used in the 1990 study may not capture well the constructs measured by the full scales. Therefore, in May 1991, a year after mailing the whole twin sample, a second questionnaire containing only the complete IPSM, PBI and IBM was mailed to a subsample of 100 twin pairs. The subsample was chosen at random from the 2000 or so pairs who had responded at that stage to the 1990 study, with the proviso that there should be equal numbers of male and female monozygotic and dizygotic twins (i.e. 25 pairs of each). After 3 months non-respondents were followed-up by telephone and/or letter. The final sample of returned questionnaires included 51 from males and 70 from females (60\% response rate overall).

\section{Statistics}

To minimize loss of data through listwise deletion, 13 missing item responses in the 1990 data set (out of $36 \times 121=4356-0.3 \%$ ) were substituted with the most frequent response category. Subscale scores for both the shortened and full versions of all tests were calculated by summing items. From the 1991 questionnaires scores were computed for the full scale instruments using the standard scoring procedure. Short scale scores were also computed from the 1991 data using the items comprising the shortened version. From the 1990 questionnaires only short scale scores were available.

Pearson correlation coefficients were calculated for tests scores obtained from the shortened and full length versions of the instruments in 1991 and the shortened version used in 1990. Separate correlations were also carried out for male and female respondents to examine whether sex differences were evident. Finally, the intercorrelations between the IPSM, PBI and IBM within each time period were compared to determine whether the nature of the relationship between the full version tests was duplicated in the shortened versions.

\section{RESUITS}

A total of $121 \mathrm{Ss}(42.1 \%$ males) completed both the full and shortened versions of the IPSM, PBI and IBM. Mean scores obtained by the twin $S$ s on the full length instruments are shown in Table 1 together with the normative data reported by the authors of the original instruments. The twin $S$ s did not differ significantly from those used in the normative studies.

Table 2 shows the correlation coefficients berween the IPSM. PBI and IBM scores using both the shortened and full versions in 1991 and the shortened version in 1990. Correlations between the shortened and full versions in 1991 were generally high $(r=0.82-0.99)$ with the exception of the timidity subscale on the IPSM. The lower correlation for the latter scale $(r=0.64)$ suggests the shortened subscale does not fully capture this construct.

Correlations between the shortened versions of the tests in 1990 and 1991 offer an indication of stability of constructs over time. The coefficients were moderate for the IPSM $(r=0.47-0.55)$ except again for the fragile inner-self subscale $(r=0.18)$. Correlations between the shortened PBI scales were slightly higher $(r=0.55-0.66)$, as was the correlation between the shortened IBM's care subscale $(r=0.58)$. Correlations between the 1990 shortened scales with their full-length 1991 counterparts were very similar. Coefficients were slightly lower for the IPSM $(r=0.31-0.50)$, comparable or siightly higher for the PBI $(r=0.61-0.70)$. and the same for the IBM $(r=0.58)$.

Pearson correiations were calculated separately for maies and females. Results were generally consistent with those obtained by the full sample on most subscale scores. However, 1-year 
Table 1. Mean scores of the study sample versus normative data for the full iength 1991 IPSM. PBI and IBM

\begin{tabular}{lcc}
\hline & $\begin{array}{c}\text { Study sample } \\
\text { (twins) } \\
\text { Mean }\end{array}$ & $\begin{array}{c}\text { Normative data } \\
\text { Mean }\end{array}$ \\
\hline IPSM & 19.3 & 18.2 \\
Interpersonal awareness & 26.1 & 26.7 \\
Need for approvai & 17.3 & 17.0 \\
Separation anxiet: & 20.4 & 21.2 \\
Timidity & 9.0 & 8.9 \\
Fragile inner-self & 91.9 & 92.0 \\
IPSM total & & 22.9 \\
PBI/Father & 21.7 & 11.9 \\
Care & 13.5 & \\
Overprotection & & 26.8 \\
PBI/Mother & 28.1 & 14.7 \\
Care & 14.8 & \\
Overprotection & & 28.1 \\
IBM & 28.8 & 11.9 \\
Care & 8.7 & \\
Control & & \\
\hline
\end{tabular}

stabilities for males on the PBI care dimension and the IBM care subscale were notably low $(r=0.05-0.25)$.

The interrelationship between the IPSM, PBI and IBM within each time period was also examined. Previous studies (Boyce et ai., 1991) have shown the full versions of the IPSM, PBI and IBM to have low intercorrelations, suggesting the tests are measuring constructs independent of each other. As can be seen in Table 3(a) correlation coefficients between the full version IPSM, $\mathrm{PBI}$ and IBM scores used in the 1991 study were low, ranging from $r=0.06-0.37$. Intercorrelations between scores obtained on the shortened versions used in the 1990 study, shown in Table 3(b), were also low $(r=0.01-0.24)$, supporting a similar relationship between the scales.

As some of the scales used in 1990 consisted of binary response categories, and, overall, responses to many items had skewed distributions, polychoric and polyserial correlations were calculated using PRELIS (Jöreskog \& Sörbom, 1988). The resulting correlations differed little from those in Tables 2 and 3 , and in no case more than 0.1 . We also examined the internal reliability of the three

Table 2. Correlation coefficients between the full length and shortened IPSM, PBI and IBM

\begin{tabular}{|c|c|c|c|c|c|c|}
\hline & \multicolumn{2}{|c|}{ Shor:'91 vs Long'91 } & \multicolumn{2}{|c|}{ Short'90 vs Short'9l } & \multicolumn{2}{|c|}{ Short'90 vs Long'9! } \\
\hline & $\wedge$ & $r$ & $N$ & $r$ & $N$ & $r$ \\
\hline \multicolumn{7}{|l|}{ IPSM } \\
\hline Interpersonal awareness & 116 & 0.95 & 118 & 0.47 & 116 & 0.42 \\
\hline Separation anxiety & 118 & 0.90 & 119 & 0.47 & 118 & 0.39 \\
\hline Timidity & 119 & 0.64 & 121 & 0.53 & 119 & 0.35 \\
\hline Fragile inner-self & 120 & 0.82 & 120 & 0.18 & 120 & 0.31 \\
\hline IPSM tota] & 111 & 0.92 & 116 & 0.55 & 111 & 0.50 \\
\hline \multicolumn{7}{|l|}{ PBI/Father } \\
\hline Care & $10 \Sigma$ & 0.94 & 107 & 0.66 & 103 & 0.67 \\
\hline Overprotection & 101 & 0.89 & 103 & 0.65 & 99 & 0.62 \\
\hline \multicolumn{7}{|l|}{ PBI/Mother } \\
\hline Care & 110 & 0.93 & 111 & 0.55 & 108 & 0.61 \\
\hline Overprotection & 113 & 0.91 & 114 & 0.63 & 111 & 0.70 \\
\hline \multicolumn{7}{|l|}{ IBM } \\
\hline Care & $8 \bar{a}$ & 0.99 & 35 & 0.58 & 35 & 0.58 \\
\hline
\end{tabular}

Table 3. Intercortelations between the IPSM. PBI and IBM

\begin{tabular}{|c|c|c|c|c|c|}
\hline & \multicolumn{2}{|c|}{ PBI care } & \multicolumn{2}{|c|}{ PBI overprotection } & \multirow[b]{2}{*}{ IBM care } \\
\hline & Father & Mothe: & Fathe: & Mother & \\
\hline \multicolumn{6}{|l|}{ (a) Full scale. 1991 data" } \\
\hline IPSM total & -0.20 & -0.26 & 0.37 & 0.33 & -0.17 \\
\hline $\begin{array}{l}\text { IBM care } \\
\text { (b) Shortened scale } 1990 \text { datat }\end{array}$ & 0.15 & 0.06 & -0.15 & -0.08 & \\
\hline IPSM tota! & -0.24 & -0.07 & 0.13 & 0.01 & 0.07 \\
\hline IBM care & 0.03 & $-0.0^{-}$ & -0.06 & -0.16 & \\
\hline
\end{tabular}

Correlations with IBM care: ${ }^{*} n=76-75$. all other correiations $n=99-105$ : ${ }^{\mathrm{t}} n=37-39$. ali other correlations $n=116-119$ 


\begin{tabular}{|c|c|c|c|}
\hline & Long $9 !$ & Short 91 & Shor: 90 \\
\hline \multicolumn{4}{|l|}{ :PSM } \\
\hline Interpersonal awareness & 0.85 & 0.83 & 0.66 \\
\hline Separation anxuety & 0.81 &. .7 & $0 .-9$ \\
\hline Timidity & $0.8 \mathrm{i}$ & 0.46 & 0.71 \\
\hline Fragile inner-seif & 0.85 & 0.94 & 0.48 \\
\hline IPSM totaí & $0.92^{2}$ & 0.83 & 0.7 \\
\hline \multicolumn{4}{|l|}{ PBI Father } \\
\hline Care & 0.94 & 0.83 & 0.79 \\
\hline Overprotection & 0.91 & 0.79 & $0.7 ?$ \\
\hline \multicolumn{4}{|l|}{ PBI/Mother } \\
\hline Care & 0.95 & 0.87 & 0.74 \\
\hline Overprotection & 0.92 & 0.83 & 0.73 \\
\hline \multicolumn{4}{|l|}{ IBM } \\
\hline Care & 0.96 & 0.96 & 0.96 \\
\hline
\end{tabular}

test versions by using the polychoric correlation matrices of item responses to calcuiate Cronbach's alpha. These results are displayed in Table 4. The alphas for the full length 1991 IPSM, PBI and IBM were all high ( $\alpha=0.81-0.96)$, consistent with figures reported by others with these instruments (Boyce \& Parker, 1989; Parker et al., 1979; Wilheim \& Parker, 1988). Cronbach's alphas for the shortened 1991 and 1990 scaies were only slightly lower $(\alpha=0.66-0.96)$. with the exception of the timidity subscale on the shortened 1991 test $(\alpha=0.46)$, and the fragile inner-self subscale on the shortened 1990 test $(\alpha=0.48)$.

\section{DISCUSSION}

In this study we compared full length and shortened versions of three questionnaires which measure possible vuinerability factors to neurotic illness. A sample of twin $S$ s compieted shortened versions of the IPSM, PBI and IBM in 1990. and full length versions of the same instruments in 1991. The twins were not a random sample but the data obtained were very similar to the normative results reported by the authors of the original instruments (Parker et al., 1979: Wilhelm \& Parker, 1988; Boyce \& Parker, 1989).

The internal reiiabilities of the shortened and full length IPSM. PBI and IBM indicated only a slight reduction in the shortened scales in most cases. With the possible exception of the IPSM timidity and fragile inner-self subscales the shortened versions appeared valid approximations of the same interpersonal constructs captured by the full scale versions.

Comparisons between the shortened and full length tests focused on the effects of three main factors. The first was the number of items used in the two versions. The high correlations between the shortened and full length scales within the 1991 study were not surprising given their interdependence. Nonetheless, they indicate that while information is lost with the reduction of items, overall the correlations remain good and some items can be dropped.

The shortened 1990 tests included the same reduced number of items as the shortened 1991 version but the 1990 test items inciuded some changes in wording and scoring. This second factor would have contributed to the lower correlations between the shortened 1990 and 1991 data. In addition. the comparisons between the 1990 and 1991 versions included the further effect of time. In a study by Wilheim and Parker (1990) they reported stability coefficients over 5 years of $0.67-0.82$ for the PBI and $0.49-0.50$ for the IBM. The present results are over a shorter period but àre consistent with these figures. The correlations between the 1990 and 1991 PBI scores were slightly higher, perhaps because recollections of early parental attachments are likely to be more stable over time. In contrast. intimate relationships rated on the IB.M are more likely to change. The partner. or the quality of the relationship. being rated in 1990 and 1991 may have been different. In addition. the instructions used to deñne 'partner' in the swo studies was not the same. In the 1990 study 'partner' referred to spouse or de facto partner. while in 1991 it included any intimate person. This broader definition explains. in part. the increase in the number of people completing the IBM from 39 in 1990 to 79 in 1991. bearing in mind that the sample was only 
aged $18-26$ years - a time when intimate relationships are being formed. The moderate correlations obtained on the IPSM subscales ove: the 1 year period raise questions about the stability of the underlying personality trai:. and suggest the instrument may be measuring a state component as well. This is consisten: with an eariie: study demonstrating a state effect on the IPSM (Boyce \& Parker. 1989).

Comparisons between the shortened 1990 tests and the full length 1991 versions incorporated a difference in the number of items as well as the differences in wording, scoring and change over time. The correiations obtained between these versions were similar to those obtained between the shortened 1990 and shortened 1991 tests suggesting that the extra items added little.

The correlations involving the shortened IPSM subscales were considerably lower than those for the shortened PBI and IBM. The presentation of items in the shortened IPSM in the 1990 study was significantly altered from the original format. The 12 selected items were reworded from statements into questions and the 4 point response set was replaced by a yes/no option. In comparing the internal reliabiiities of the shortened 1990 and 1991 IPSM scales these changes may have contributed to the slight reduction in alphas on the interpersonal awareness subscale, but such an effect was not apparent on the separation anxiety subscale. The inconsistency of Cronbach's alphas for the shortened 1990 and 1991 timidity and fragile inner-self subscales may reflect the fact that only two items comprised each scale, making these particular subscales more prone to sampling fluctuations.

By contrast. the 7 items used in the shortened 1990 PBI were presented essentially verbatim. The 4-point response set was retained, although the response labels were slightly rephrased. The similar internal reliability results within the shortened 1990 and 1991 versions, particularly for fathers, and the relatively higher Pearson correlations between the 3 test versions, suggest these minor changes were of little significance.

The shortened IBM was the most intact of the 3 instruments. It comprised 10 out of the 12 items of the full version IBM care subscale. and used the same 4-point response set. The identical internal reliability results of the 3 test versions indicate the 2 omitted items can safely be dropped. In addition, the modest correlations found over 1 year can more confidently be interpreted as changes in intimate relationships over time rather than changes due to questionnaire format. In contrast, for the IPSM it is not possibie to determine whether the poorer correlations were the result of a state effect. a tesi-retest artefact (Jorm. Duncan-Jones \& Scott, 1989), or the result of the changes to item wording and scoring.

Separate comparison of scores for males and females generally indicated similar correlations between test versions and over time. However. males showed significant changes in responses on the PBI care and IBM care subscales over the 1 year period. Some of the variation on the IBM may be accounted for by changed intimate reiationships, but the PBI result is more difficult to interpret, particularly given the sample size.

While the modest correlations between the 1990 and 1991 IPSM, PBI and IBM may question the stability of the measures. the relationship between the tests remained constant. The low intercorrelations between the 1991 full length IPSM, PBI and IBM were mirrored in the shortened 1990 test versions. and indicate that the independence of tests was maintained. This finding is significant as the interrelationship between risk variables is often the focus of studies developing causal models and the use of these shorened versions would not compromise such studies. It would be unwise. however. to assume the full scale IPSM. PBI and IBM instruments are redundant. The shortened versions yield simpiiffed measures which would be inappropriate for use in research on inpatient psychiatric populations or community based studies. We have confidence nonetheless that the shortenec IPSM. PBI and IBM scaies used in the 1990 questionnaire study are valid. as judged against the full instruments. The sacrifice in internal reliability is more than matched by the reduction in iength. Wie furthe: sugges: that these shortened versions may find use in other areas of psychiatric epidemiology: bu: hasten to add greater attention be given to some of the methodological issues raised above.

Acknowledgements - The research was supportec oy grants from the Nationa! Health and Medical Research Council of Austraila (NH\&.MRC:. ine LS Nationa: Insutule of Aiconoilsm. anc Aiconol Abuse (NIAAA-AA07535) anc Wentworth Area Heaith Serise. Twins inciudec in the sampie are from the Austraiian NH\&MRC Twin Registry director Dr Johr. 
Hopper. whom we thank for cooperation. We also thank Ann Eldridge, Sue Mason and Olivia Zheng ior assistance irith data collection. and John Pearson and the late Ulrich Kehren for data management.

\section{REFERENCES}

Alloway. R. \& Bebbington. P. (1987). The buffer theory of social support-a review of the literature. Psychoiogical Medicine. $17,91-108$.

Andrews. G.. Stewart, G., Allen, R. \& Henderson, A. S. (1990). The genetics of six neurotic disorders-a twin study. Joumal of Affective Disorders. 19. 23-29.

Bowlby, J. (1988). Developmental psychiatry comes of age. American Journal of Psychiatry, 145. 1-10.

Boyce, P. M. \& Parker. G. (1989). Development of a scale to measure interpersonal sensitivity. Australian and New Zeaiand Journal of Psychiatry, 23, 341-351.

Boyce. P. M.. Hickie, I. \& Parker, G. (1991). Parents, partners or personaiity? Risk factors for post-natal depression. Journal of Affective Disorders, 21, 245-255.

Boyce, P. M., Parker, G., Hickie, I.. Wilheim, K., Brodaty, H. \& Mitchell, P. (1990). Personality difierences between remitted melancholic and non-melancholic depressives. American Journal of Psychiatry, 147, 1476-1482.

Cadoret. R. J., O'Gorman. T. W., Heywood. E. \& Troughton. E. (1985). Genetic and environmental factors in major depression. Journal of Affective Disorders, 9, 155-164.

Eysenck, S. B. G.. Eysenck. H. J. \& Barrett, P. (1985). A revised version of the Psychoticism scale. Personality and Individual Differences, 6. 21-29.

Henderson, S., Duncan-Jones, P., McAuley, H. \& Ritchie, K. (1978). The patient's primary group. British Journal of Psychiatry, 132, 74-86.

Hickie, I., Wilheim, K., Parker, G., Boyce, P., Hadzi-Pavlovic, D., Brodaty, H. \& Mitchell. P. (1990). Perceived dysfunctional intimate relationships: a specific association with the non-melancholic depressive subtype. Journal of Affective Disorders, 19. 99-107.

Jardine, R., Martin. N. G. \& Henderson. A. S. (1984). Genetic covariation between neuroticism and the symptoms of anxiety and depression. Genetic Epidemiology, 1, 89-107.

Jōreskog, K. G. \& Sörbom, D. (1988). PRELIS-a program for multivariate data screening and data summarisation. A preprocessor for LISREL (2nd Edn). Mooresville, IN: Scientific Software Inc.

Jorm, A. F., Dunan-Jones, P. \& Scott, R. (1989). An analysis of the re-test artefact in longitudinal studies of psychiatric symptoms and personality. Psychological Medicine, 19, 487-493.

Kendler, K. S., Neale, M. C., Kessler, R. C., Heath. A. C. \& Eaves, L. J. (1992). A population-based twin study of major depression in women-the impact of varying definitions of illness. Archives of General Psychiatry, 49. $257-266$.

Martin, N. G., Jardine. R., Andrews, G. \& Heath, A. C. (1988). Anxiety disorders and neuroticism: are there genetic factors specific to panic? Acta Psychiatrica Scandinavica, 77, 698-706.

Parker, G. (1983a). Parental 'affectionless control' as an antecedent to adult depression: a risk factor deiineated. Archives of General Psyciniatry, 40, 956-960.

Parker, G. (1983b). Parental overprotection: A risk factor in psychosocial development. Sydney: Grune \& Stratton.

Parker, G.. Kiloh. L. \& Hayward, L. (1987). Parental representations of neurotic and endogenous depressives. Journal of Affective Disorders, 13, 75-82.

Parker, G., Tupling, H. \& Brown, L. B. (1979). A Parental Bonding Instrument. British Journal of Medical Psychology, $521-10$.

Plantes, M. M., Prusoff. B. A.. Brennan, J. \& Parker, G. (1988). Parental representations ố depressed outpatients from a U.S.A. sampie. Journal of Affective Disorders, 15, 149-155.

Wilhelm, K. \& Parker, G. (1988). The development of a measure of intimate bonds. Psychological Medicine, 18. $225-234$.

Wilhelm, K. \& Parker, G. (1990). Reliability of the Parental Bonding Instrument and Intimate Bond Measure scales. Australian and New Zealand Journal of Psychiatry, 24, 199-202. 